\title{
Sellar Atypical Teratoid Rhabdoid Tumor (Atrt) In An Adult: A Case Report and Review of The Literature
}

\author{
Daniel Barsky*1, Uri P Hadelsberg ${ }^{2}$, Lior Gonen² and Nevo Margalit ${ }^{2}$ \\ ${ }^{1}$ School of medicine, Hebrew University of Jerusalem, Israel \\ ${ }^{2}$ Department of Neurosurgery, Shaare Tzedek medical center, Jerusalem, Israel
}

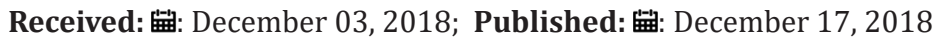

*Corresponding author: Daniel Barsky, School of medicine, Hebrew University of Jerusalem, Israel

\begin{abstract}
Background: Atypical teratoid rhabdoid tumor (ATRT) is a rare type of central nervous system (CNS) tumor, which is seen mostly in the pediatric population. However, there are a few dozen of cases reported in adults. This entity was first differentiated from a Wilm's tumor in 1978. Since then much progress has been made in understanding the pathophysiology of this disease, but only slight progression has been done in terms of treatment and prognosis, thus ATRT remains a high mortality-disease with grim prognosis and outcome.
\end{abstract}

Case Description: 54 year-old female, presented with symptoms concurrent of a pituitary macroadenoma (e.g. temporal field vision loss, hyperprolactinemia) which was found out to be a tumor of ATRT characteristics on pathology.

Conclusion: ATRT is a rare entity, but should be kept in mind since it has a poor prognosis. Moreover, considering the grim prognosis, and the rarity of the disease, once ATRT diagnosis was made, it should be treated by a multidisciplinary team composed with surgeons, oncologists and radiation oncologists.

Keywords: ATRT; Macroadenoma; Trans-sphenoidal surgery

Abbreviations: ATRT: Atypical Teratoid Rhabdoid Tumor; CNS: Central Nervous System; CT: Computed Tomography; MRI: Magnetic Resonance Imaging; AT/RT: Atypical Teratoid/Rhabdoid Tumor: ICU: Intensive care Unit; DI: Diabetes Insipidus; LMD: Leptomeningeal Dissemination; EMA: Epithelial Membrane Antigen; GFAP: Glial Fibrillary Acidic Protein; TSG: Tumor Suppressor Gene

\section{Introduction}

Atypical teratoid rhabdoid tumor (ATRT) is a rare type of central nervous system (CNS) tumor, composed of rhabdoid cells. It effects mostly children younger than three years of age. The location of the tumor is often supratentorial, but it can be found in all parts of the CNS [1], this tumor is characterized with a high mortality rate [1,2]. It was first described by Beckwith and Palmer as a variant of Wilms tumor in 1978 [3]. There are few reports about ATRT in adults, but it is less common in that population [4-6]. In a review made by Sinha et al. the majority of the tumors in adults were located within the lobes of the cerebrum[6]. The rarity of the entity and its variable presentation make the diagnosis challenging, and even with the use of radiology and pathology ATRT is still misdiagnosed [7]. We report a case of a primary sellar ATRT in a 54 year-old female, which was presented and treated as a pituitary macroadenoma until the pathological diagnosis was made as an ATRT tumor.

\section{Case Report}

\section{History and Examination}

A 54 year-old right handed female, without any significant past medical history, was admitted with a 3 month history of progressing fatigue, nausea, shivers, and weight loss. Headaches, blurred vision or galactorrhea were absent. On physical examination the patient was fully oriented, with a left side 3rd cranial nerve palsy displaying a left side ptosis and slight left pupil dilatation. Both Pupils were reactive to light with no limits on eye movement. The rest of the physical exam was insignificant. A neuro-opthalmologic examination revealed bilateral visual accuracy of 20/30, left eye temporal field loss and enlargement of the blind spot in the right eye. Laboratory analysis showed hyperprolactinemia (54ng/ $\mathrm{ml}$ ), and low cortisol levels $(2.6 \mathrm{mcg} / \mathrm{dL})$. Due to these values, a 5 miligram dose of prednisone twice daily was initiated. A synacthen 
test was preformed which revealed low levels of cortisol and free $\mathrm{T} 4$, therefore treatment with daily thyroxine $50 \mathrm{mcg}$ was started as well. The clinical findings were suitable with a pituitary adenoma, with stalk effect.

Computed tomography (CT) imaging was obtained which revealed a sellar and suprasellar mass of $1.6 * 1.1 * 2.4$ centimeters $(\mathrm{cm})$ in size, with $1.6 \mathrm{~cm}$ extension of the suprasellar cistern, with homogenous enhancement. Brain magnetic resonance imaging (MRI) was preformed which further delineated a sellar and suprasellar mass which cannot be separated from the hypophysis, with a maximal height of $25 \mathrm{~mm}$, and a small amount of edema noted (Figures 1-3). These radiological findings changed our differential diagnosis and the option of non-pituitary tumors was discussed. The patient was planned for an endoscopic trans-sphenoidal (TSS) operation.

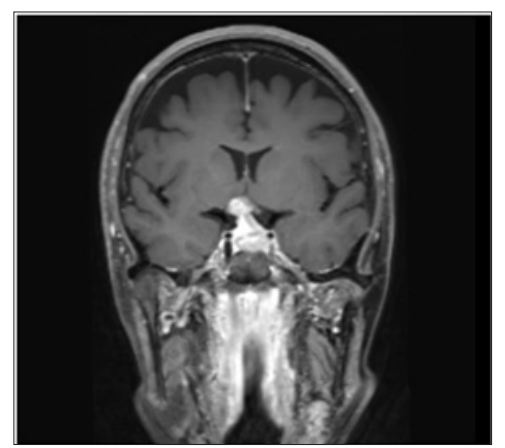

Figure 1: T1 coronal demonstrating a SOL suspected of a pituitary adenoma which was later found out to be an ATRT tumor.

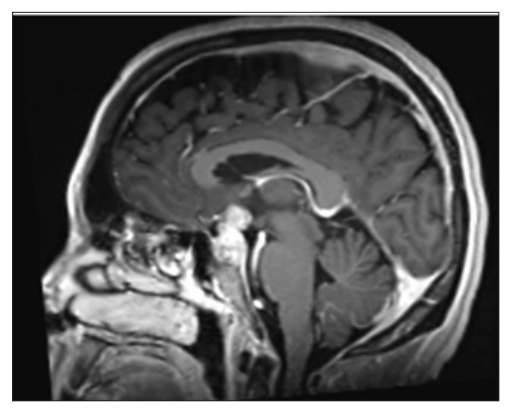

Figure 2: A T1 sagittal MRI further demonstrating this suprasellar tumor.

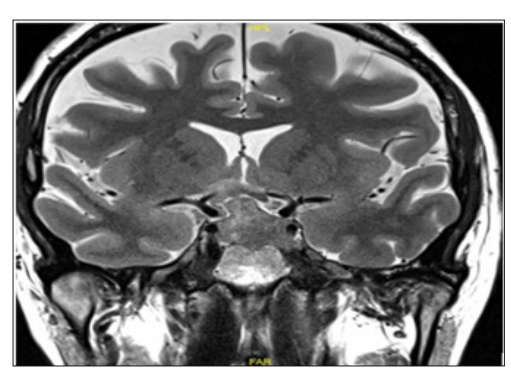

Figure 3: This is a MRI taken 2 weeks after presentation. The patient's vision deteriorated. This coronal T2 MRI demonstrates edema in the right optic tract.

\section{Surgery}

An endoscopic endonasal transsphenoidal approach was performed. The sellar region was mildly enlarged. The tumor was dissected from the right optic nerve and optic chiasm. During surgery the tumor had an atypical consistency and the separation from the optic apparatus was difficult. The sellar defect was reconstructed with abdominal fat and a naso- septal flap. The patient was extubated with no new neurological deficits and transferred to the intensive care unit for further treatment. A CT performed postoperatively demonstrated subtotal resection of the tumor and no acute findings, MRI follow up demonstrated a small residual tumor (Figures $4 \& 5$ ).

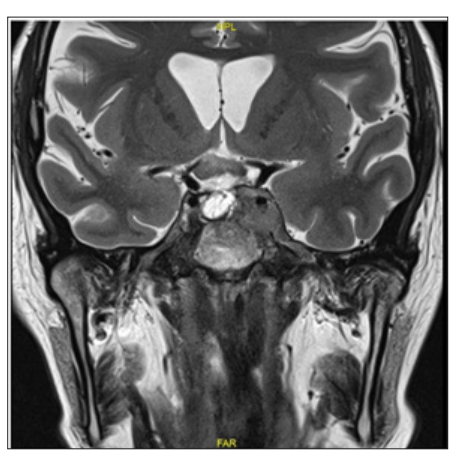

Figure 4: A T2 coronal MRI showing the chiasm clean of tumor with CSF between the optic chiasm and the sella turcica.

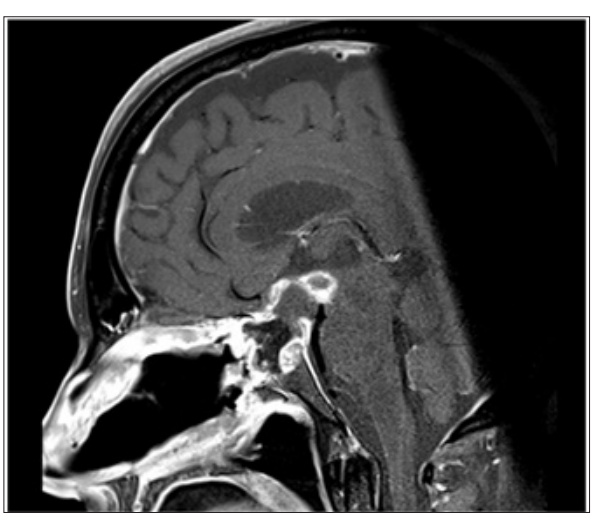

Figure 5: Sagittal T1 MRI done after surgery displaying an adequate amount of resection to the lesion with a small amount of postoperative bleed.

\section{Pathology}

The lesion was sent to the histopathological analysis. Multiple irregular fragments of tissue were received; the largest part being1 $\mathrm{cm}$ in diameter. The histopathological analysis demonstrated big cells with vesicular nuclei, prominent nucleoli and a little amount of cytoplasm, forming sheets of tumorous cells infiltrating the hypophysal tissue, mitoses and necrosis were also seen. Tumor cells were positively stained for p51, p53 and S-100. There was negative staining for pituitary hormones and chromogranin. MIB1 proliferation index showed $50 \%$ of cells. Staining for Pit1, somatostatin receptor, CK7, Ck20, CK18, Oct4, TTF1, LCA, CD3, 
CD20, Melan A, PanCK were all negative. Immunohistochemical staining for INI1 was negative in the neoplastic cells. Diagnosis of a sellar atypical teratoid/rhabdoid tumor (AT/RT) was made.

\section{Postoperative Course}

After surgery the patient was admitted to the intensive care unit (ICU) for monitoring and antibiotic treatment due to suspected meningitis. During her stay in the ICU diabetes insipidus (DI) was noted and treated by intravenous (IV) desmopressin. Due to the patient's final diagnosis of ATRT, she underwent spinal MRI and total body CT which did not demonstrate any metastatic spread. The patient was discharged in good condition for further oncological treatment, while she is autonomously walking, drinking and eating, talking fluently, but suffering from mild fatigue.

\section{Discussion}

ATRTs mostly affect children less than 3 years of age, and constitute $1-2 \%$ of pediatric tumors. It is the most common type of CNS tumor in children less than 6 months of age $[7,8]$. At the time of diagnosis $20 \%$ of patients have a disseminated disease, mostly present as leptomeningeal dissemination (LMD). Several studies demonstrate a median overall survival in children of 6 months $[1,8]$. Presenting symptoms can be non-specific such as lethargy, vomiting andshead ache [1,9]. There are only few dozens of ATRT cases reported in adults [10], which differ by their location- While in children the majority of tumors are located in the posterior fossa, in adults the majority of tumors are supratentorial. Other locations such as the sella turcica, as in this patient, are less common $[1,6]$. The clinical presentation in adults is varied: Vomiting and ataxia, limb numbness, prolactinoma, cauda equina syndrome neck and back pain were previously reported [5,6,10-12]. Because of nonspecific clinical signs, advanced imaging must be obtained as soon as possible. On CT imaging, ATRT lesions are shown to be hyperdense compared to the surrounding gray matter, probably due to the high cellular density within the tumor $[13,14]$.

Brain MRI findings on $\mathrm{T} 1$ and $\mathrm{T} 2$ weighted imaging varied widely, and the findings are not consistent. In the majority of ATRTs demonstrate areas of hemorrhage, and cystic and necrotic areas in the lesion, and in some of the tumors edema can be seen $[13,14]$. On histopathology ATRT demonstrates dense round small blue cells which resemble to those in PNET [15], along with a rhabdoid component composed of medium-large cells with eccentric vesicular nuclei, prominent nucleolus and eosinophilic cytoplasm as seen in our case. Rhabdoid cells are often immunopositive for vimentin, epithelial membrane antigen (EMA), smooth muscle actin and sometimes neuroectodermal markers such as glial fibrillary acidic protein (GFAP) and others $[9,15]$. ATRTs are highly mitotic with prominent necrosis, and MIB1 mitotic index can be high [9]. One of the major factors implying the diagnosis of ATRT is mutation or loss of expression of INI1 protein [6,9,15-17], which can be proven by immunochemical staining, as seen in our case. The loss of expression of INI1 might be due to chromosomal deletion as well as due to DNA hypermethylation [17].
hSNF5/INI1 is a tumor suppressor gene (TSG) found on chromosome 22q11.2, this gene encodes for INI1 which is a member of chromatin remodeling SWI/SNF multiprotein [18]. It was demonstrated that SWI/SNF complex is required for RBmediated inhibition of proliferation, which is a well-studied TSG [17]. This mutation is considered as a main event in forming of ATRT [19]. Recent evidence suggests that adult sellar region ATRTs have methylation patterns similar to those seen in pediatric ATRTMYC subgroup [20].

Because adult onset of ATRT is highly uncommon, there are no official guidelines, nor studies that compare different types of treatment in adults. Current treatment options are surgery, chemotherapy and radiotherapy. In a study lead by Lau et al. 174 ATRT patients were analyzed. $98.5 \%$ of them were 19 yearold and less. This study suggested an overall cumulative survival higher when tumors were treated with radiotherapy and surgery compared to surgery alone [21]. A study lead by Buscarillo et al. included 144 patients with ATRT. Only 3 of them were adults. The overall survival was higher in patients receiving radiotherapy [2].

Hilden et al. compared outcomes of several treatment strategies in 42 pediatric cases of ATRT showing higher rates of free survival and better overall survival in patients who underwent total gross resection compared to a partial resection, albeit all patients receiving adjuvant therapy [22]. Another study, done by Wetmore et al. had 4 pediatric patients with new site progressive ATRT. During the study, the effect of alisertib- aurora kinase A (AURKA) inhibitor was measured. AURKA encodes cell cycle associated serine/ threonine kinase that regulates mitosis, and it is up-regulated in the loss of INI1, as occurs in ATRT. Inhibition of AURKA by alisertib causes mitotic delays followed by cell death [23]. It showed disease stabilization, or decrease in tumor size, in all patients after disease recurrence [23]. Other therapeutic strategies and compounds are still being tested, and are now recruiting patients to their clinical trials [19] with results yet to be published.

\section{Conclusion}

Sellar region ATRT are rare and pose a diagnostic and therapeutic challenge. ATRT has a varied clinical presentation, nonspecific radiological findings and complex pathophysiology. Because theses tumors are rare and have grim prognosis, a multidisciplinary approach including a surgeon, oncologist and radiation oncologist should be involved in the treatment to increases survival rates. Further research referring to treatment options and outcomes is warranted for all age groups harboring this type of brain tumor.

\section{Declarations}

Funding: This research did not receive any specific grant from funding agencies in the public, commercial, or not-for-profit sectors.

\section{References}

1. Rorke LB, Packer RJ, Biegel JA (1996) Central nervous system atypical teratoid/rhabdoid tumors of infancy and childhood: definition of an entity. J Neurosurg 85: 56-65. 
2. Buscariollo DL, Park HS, Roberts KB, Yu JB (2012) Survival outcomes in atypical teratoid rhabdoid tumor for patients undergoing radiotherapy in a Surveillance, Epidemiology, and End Results analysis. Cancer 118(17): 4212-4219.

3. Beckwith JB, Palmer NF (1978) Histopathology and prognosis of Wilms tumors: results from the First National Wilms' Tumor Study. Cancer 41: 1937-1948.

4. Las Heras F, Pritzker KPH (2010) Adult variant of atypical teratoid/ rhabdoid tumor: Immunohistochemical and ultrastructural confirmation of a rare tumor in the sella tursica. Pathol -Res Pract 206: 788-791.

5. Zarovnaya EL, Pallatroni HF, Hug EB, Ball PA, Cromwell LD, et al. (2007) Atypical teratoid/rhabdoid tumor of the spine in an adult: Case report and review of the literature. J Neurooncol 84: 49-55.

6. Sinha P, Ahmad M, Varghese A, Parekh T, Ismail A, et al. (2015) Atypical teratoid rhabdoid tumour of the spine: report of a case and literature review. Eur Spine J 24: 472-484.

7. Biswas A, Kashyap L, Kakkar A, Sarkar C, Julka PK, et al. (2016) Atypical teratoid/rhabdoid tumors: challenges and search for solutions. Cancer Manag Res 8: 115-125.

8. Udaka YT, Shayan K, Chuang NA, Crawford JR (2013) Atypical presentation of atypical teratoid rhabdoid tumor in a child. Case Rep Oncol Med pp. 815923.

9. Biswas A, Goyal S, Puri T, Das P, Sarkar C, et al. (2009) Atypical teratoid rhabdoid tumor of the brain: case series and review of literature. Childs Nerv Syst, pp. 25.

10. Takahashi K, Nishihara H, Katoh M, Yoshinaga T, Mahabir R, et al. (2011) A case of atypical teratoid/rhabdoid tumor in an adult, with long survival. Brain Tumor Pathol 28: 71-76.

11. Barresi V, Lionti S, Raso A, Esposito F, Cannavò S, et al. (2017) Pituitary atypical teratoid rhabdoid tumor in a patient with prolactinoma: A unique description. Neuropathology 38(3): 260-267.

12. Lutterbach J, Liegibel J, Koch D, Madlinger A, Frommhold H, et al. (2001) Atypical teratoid/rhabdoid tumors in adult patients: case report and review of the literature. J Neurooncol 52: 49-56.

13. Warmuth Metz M, Bison B, Dannemann Stern E, Kortmann R, Rutkowski $S$, et al. (2008) CT and MR imaging in atypical teratoid/rhabdoid tumors of the central nervous system. Neuroradiology 50: 447-452.

\section{ISSN: 2574-1241}

DOI: $10.26717 / B J S T R .2018 .12 .002227$

Daniel Barsky. Biomed J Sci \& Tech Res

(C) (i) This work is licensed under Creative

(c) ${ }_{\mathrm{BY}}$ Commons Attribution 4.0 License

Submission Link: https://biomedres.us/submit-manuscript.php
14. Meyers SP, Khademian ZP, Biegel JA, Chuang SH, Korones DN, et al. (2006) Primary intracranial atypical teratoid/rhabdoid tumors of infancy and childhood: MRI features and patient outcomes. Am J Neuroradiol 27: 962-971.

15. Nandeesh B, Chabra M, Chand A, Rout P (2015) A clinicopathological study of atypical teratoid/rhabdoid tumor with review of the literature. Clin Cancer Investig J 4: 34.

16. Coccé MC, Lubieniecki F, Kordes U, Alderete D, Gallego MS, et al. (2011) A complex karyotype in an atypical teratoid/rhabdoid tumor: Case report and review of the literature. J Neurooncol 104: 375-80.

17. Biegel J a, Kalpana GG, Knudsen ES, Tumors TR, Packer RJ, et al. (2002) The Role of INI1 and the SWI / SNF Complex in the Development of Rhabdoid Tumors : Meeting Summary from the Workshop on Childhood Atypical Teratoid / Rhabdoid Tumors The Role of INI1 and the SWI / SNF Complex in the Development of Rhabdoid Tumors : Meetin. Cancer Res 62: 323-328.

18. Versteege I, Sévenet N, Lange J, Rousseau Merck MF, Ambros P, et al. (1998) Truncating mutations of hSNF5/INI1 in aggressive paediatric cancer. Nature 394: 203-206.

19. Frühwald MC, Biegel JA, Bourdeaut F, Roberts CWM, Chi SN, et al. (2016) Atypical teratoid/rhabdoid tumors-current concepts, advances in biology, and potential future therapies. Neuro Oncol 18: 764-778.

20. Johann PD, Bens S, Oyen F, Wagener R, Giannini C, et al. (2018) Sellar Region Atypical Teratoid / Rhabdoid Tumors ( ATRT ) in Adults Display DNA Methylation Profiles of the ATRT-MYC Subgroup 42(4): 506-511.

21. Lau CS, Mahendraraj K, Chamberlain RS (2015) Atypical teratoid rhabdoid tumors: a population-based clinical outcomes study involving 174 patients from the Surveillance, Epidemiology, and End Results database (1973-2010). Cancer Manag Res 7: 301-309.

22. Hilden JM, Meerbaum S, Burger P, Finlay J, Janss A, et al. (2004) Central nervous system atypical teratoid/rhabdoid tumor: Results of therapy in children enrolled in a registry. J Clin Oncol 22: 2877-2884.

23. Wetmore C, Boyett J, Li S, Lin T, Bendel A, et al. (2015) Alisertib is active as single agent in recurrent atypical teratoid rhabdoid tumors in 4 children. Neuro Oncol 17: 882-888.

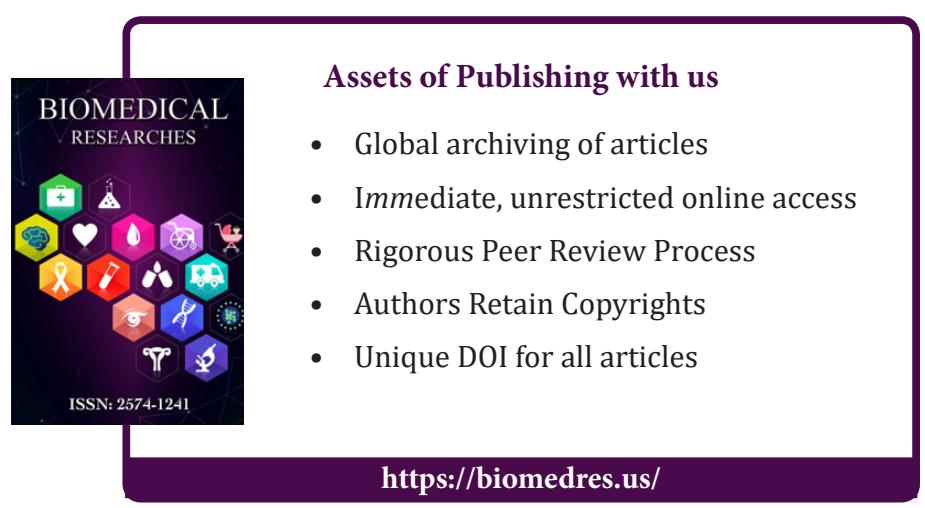

